\title{
Multi-disciplinary Approach in Management of Rhino-orbito-cerebral Mucormycosis in Resource-poor Setting in Sub-saharan Africa: A Case Report
}

\author{
Ojabo CO, ${ }^{1}$ Adekwu A, ${ }^{2}$ Ben-Ameh T. ${ }^{3}$ \\ 'Department of Ophthalmology, College of Health Sciences, Benue State University, Makurdi, Nigeria. \\ ${ }^{2}$ Department of Otorhinolaryngology, College of Health Sciences, Benue State University, Makurdi, Nigeria. \\ ${ }^{3}$ Department of Ophthalmology, Benue State University Teaching Hospital, Makurdi, Nigeria.
}

*Corresponding Author: : Ojabo CO, Department of Ophthalmology, College of Health Sciences, Benue State University, Makurdi, Nigeria.

E-amail:leojabo@yahoo.com.

\begin{abstract} Date Published: 03/04/2020

Rhino-orbito-cerebral mucormycosis (ROCM) is an uncommon acute aggressive fungal infection occurring in several immunocompromised states including diabetes which is the most common predisposing factor. In this case report we present the clinical finding, therapeutic challenges and outcome in a 44 years old male type II diabetic patient. Patient first presented to the ortorhinolaryngologist with complaints of nasal discharge, postnasal drip, cough, hyposmia, facial pains, and intermittent nasal obstruction. He had bilateral intranasal antrostomy, upon X-ray confirmation of homogenious opacity of the left maxillary antrum and mucosal thickening of the right antrum. Fungal study of the specimen revealed mucormycosis. Six months later he presented with perception of light (PL) vision in the right eye and non-perception of light (NPL) in the left eye. There was mild lid edema in the right eye. There was marked proptosis associated with external ophthalmoplegia suggestive of orbital apex syndrome. The conjunctiva was keratinized and the cornea necrotic and opaque. The left eye enucleation and nasal exploration were done. The patient objected to taking amphotericn B, the drug of choice for this condition because of his previous adverse reaction experience to the drug. Posacornazole, the second line drug could not be assessed, posing a great therapeutic challenge in the effective management of this case. The patient was later referred for neurosurgical management but was reported to have died at a tertiary health facility in Abuja.
\end{abstract} Date Accepted: $17 / 02 / 2020$

Keywords:Adverse drug reaction, Amphotericin B, Diabetes, Orbitalapex syndrome, ROCM

\section{INTRODUCTION}

$\mathrm{M}$ ucormycosis is a rare aggressive opportunistic infection caused by a filamentous fungi in the family of Mucorcea and is frequently seen in Diabetes Mellitus and other immunocompromised states. Mucormycosis is categorised as rhino-cerebral, pulmonary, cutaneous, gastrointestinal or disseminated depending on the organ affected. ${ }^{1,2,3}$ The most common form is the rhino-cerebral (40\%). This can be divided into subtypes of rhino-nasal, rhino-orbital or rhino-orbitocerebral. Rhino-orbito-cerebral mucormycosis (ROCM) is an uncommon acute and aggressive fungal infection occurring in several immunocompromised states 
including Diabetes Mellitus which is the most common predisposing factor accounting for $60-81 \%$.

Other underlying medical conditions that predispose to this infection include neutropenia, AIDS, malnutrition, burns, severe trauma, renal failure leukaemia, lymphoma and longterm steroid or immunosuppressive therapy. ${ }^{1,3,5-7}$

This infection is characterised by a rapid progression among the tissues, angioinvasion and high mortality, and represents the $3^{\text {rd }}$ most common fungal infection after candidiasis and aspagillosis. ${ }^{1-5}$ Other symptoms may include nasal congestion, rhinorrhea, postnasal drip, facial pain, chemosis, visual loss, ophthalmoplegia and proptosis. Ophthalmoplegia results from infection invading the extraocular muscles orbital space and apex during which the $3^{\text {rd }}, 4^{\text {th }}$ and $6^{\text {th }}$ cranial nerves are affected at the apex. Peripheral seventh cranial nerve paresis or palsy and hypoaesthesia of the face are often observed. $^{1,2,3,4}$ In this article, we report a case of ROCM in an immune suppressed and diabetic patient who reacted adversely to amphotericin B, the drug of choice available in our environment thus posing a great challenge in his management.

\section{CASE REPORT}

A 44 year-old male with type II Diabetes Mellitus presented to the Ophthalmology department with left periocular swelling, gradual painless axial proptosis of six months duration, total visual loss and severe visual impairment in the left and right eye respectively. He had earlier presented to the otorhinolaryngology clinic with symptoms of nasal discharge, postnasal drip, hyposmia, facial pains, and intermittent nasal obstruction. There were no ophthalmological symptoms then. Anterior rhinoscopy showed red swollen mucosa and engorged turbinares. X-ray paranasal sinuses revealed completely opacified left maxillary antrum, mucosal thickening of the right maxillary antrum and intact sinus bony walls. His haematological indices were normal and his blood sugar was brought under control. He had bilateral intranasal antrostomy and fungal study of antral specimen indicated rhinomucormycosis and was promptly commenced on IV amphotericin B which had to be discontinued after one week due to severe adverse drug reaction.

On ophthalmological examination, visual acuity was perception of light on the right and non perception of light on the left. However, on the left eye, there is lagophthalmos and proptosis of $28 \mathrm{~mm}$ as shown in Figure 1a \& Figure 1b. Extra ocular motility restricted in all fields of gaze, indicating an orbital apex syndrome. The conjunctiva was keratinised and dry, the cornea necrotic and opaque. The right eye was not proptosed $21 \mathrm{~mm}$, the ocular adnexea was normal. The anterior segment was normal. The posterior segment of the

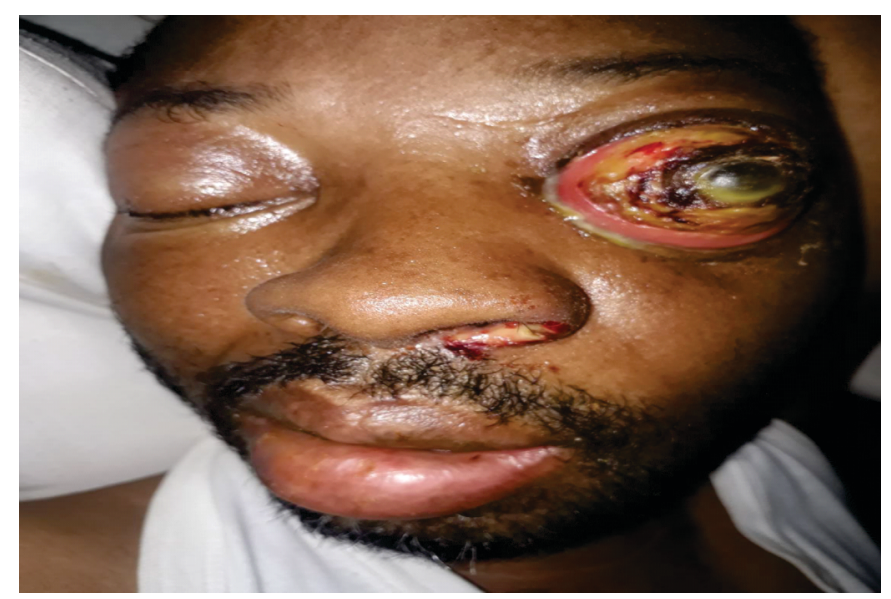

Figure 1a: Left proptosis and necrotic exposure keratopathy with facial deviation to the right

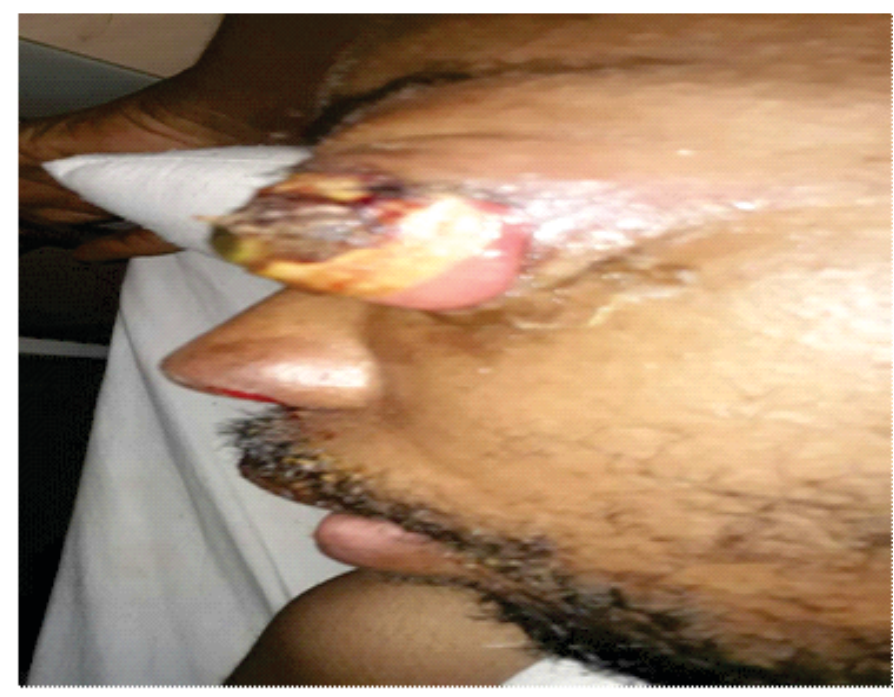

\section{Figure lb: The side view of the left proptosis}

right eye had a cup/disc ratio of 0.6. He had associated (L) facial nerve palsy with resultant facial deviation to the right.

Laboratory investigation showed a haemogram of $25 \%$. Blood chemistry showed mild hypokalamia. Blood sugar was adequately controlled on injectable insulin (Humulin) at $5.4 \mathrm{mmol}$. He was transfused with 2 units of blood raising the packed cell volume (PCV) to $33 \%$. $\mathrm{CT}$ scan revealed a mass in the left antrum, ethmoidal and 


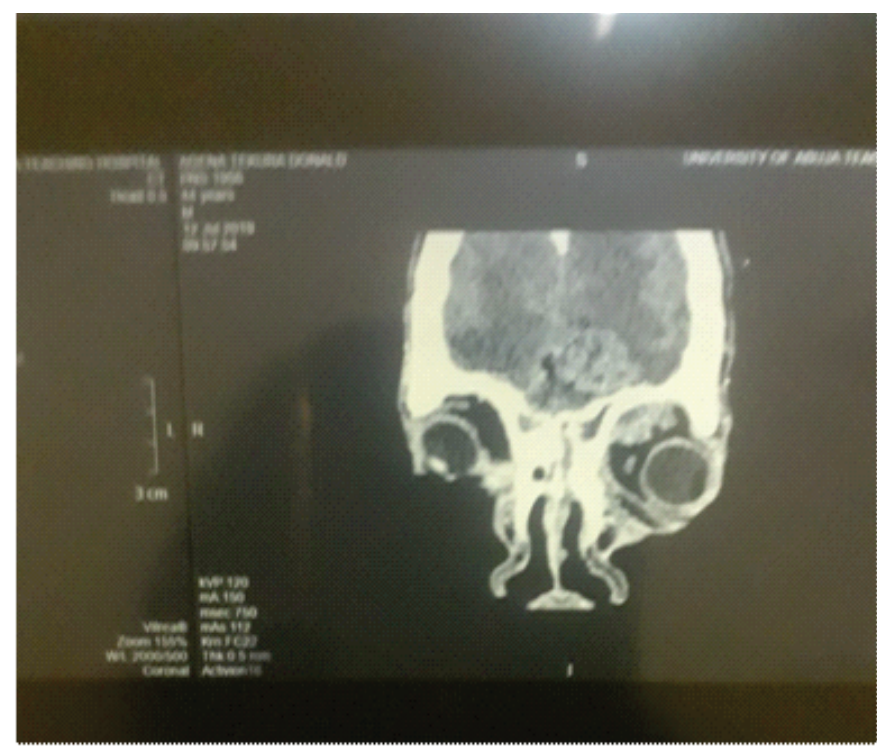

Figure 2a: CT scan showing massive invasion of the left orbit, maxillary antrum, ethmoidal and frontal sinuses.

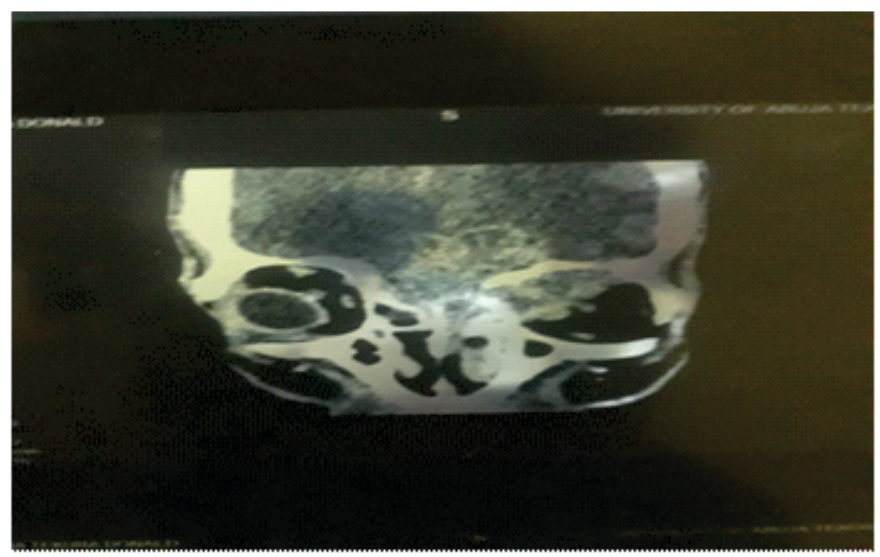

Figure 2b: CT scan showing destruction of the zygomatic bone

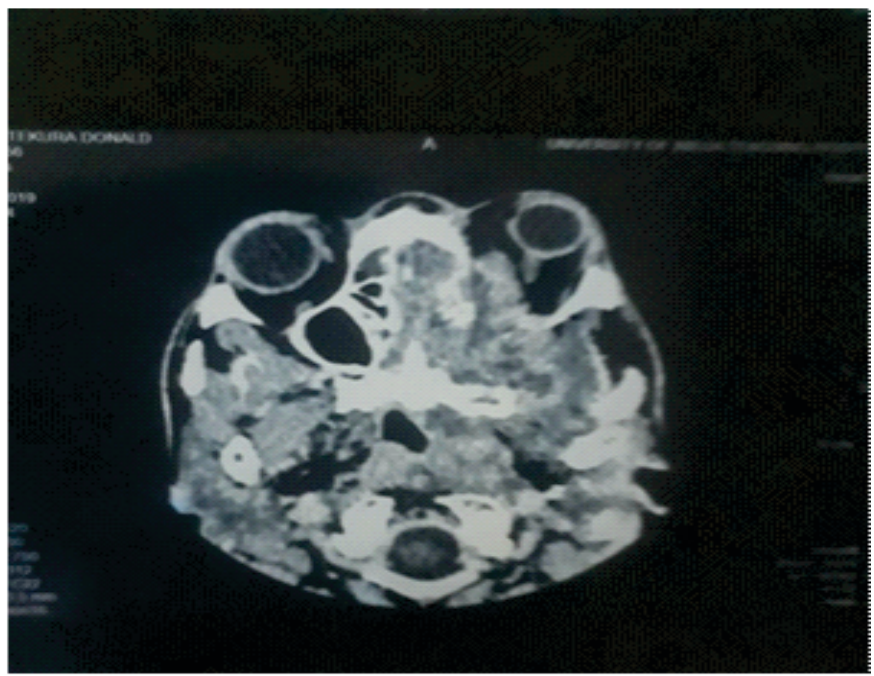

Figure 2c: CT scan showing intracranial extension to the frontal lobe frontal sinuses with destruction of the left zygomatic bone and intracranial extension into the frontal lobe as shown in figures $2 \mathrm{a}, 2 \mathrm{~b} \& 2 \mathrm{c}$.

In conjunction with Otorhinolaryngology team the patient had left enucleation and surgical debridement with removal of fungal debris in the left antrum, ethmoids and orbit. The patient was given intravenous fluconazole $200 \mathrm{mg}$ O.D, intravenous metronidazole ceftriazone and humulin.

The enucleated left eye and excised debris were sent for histology and further fungal studies. The patient objected to amphotericin B due to an earlier adverse reaction. We then referred the patient to a Neurosurgeon in another tertiary healthcare facility for further management of the cerebral component of the disease. We later got a report that the patient died.

\section{DISCUSSION}

Rhino-orbito-cerebral mucormycosis (ROCM) is a severe opportunistic fungal infection. This infection is characterized by rapid progression and high mortality and represent the third most common fungal infection after candidiasis and aspergillosis. The incidence of fungal infections is rising due to increased number of immunocompromised individuals as seen in Diabetes, AIDS, leukaemia, lymphoma and use of immunosuppressive agents. ${ }^{4,5,6}$ The most common risk factor is Diabetes Mellitus $(60-81 \%))^{7}$ Other risk factors are haematological malignancy neutropenia, pharmacological immunosuppressive agents (chemotherapy, prolong steroid usage), Diabetes Mellitus type I \& II with or without ketoacidosis. The most common underlying condition for the oral, nasal, paranasal and maxillofacial area is Diabetic Mellitus. ${ }^{8}$

The case we report is a patient who was diagnosed with Diabetes Mellitus type II of six years duration. Mucormycosis is an invasive fungal infection which has affinity for arteries and veins producing angioinvasion of the vascular walls, causing mechanical and toxic damage to the intima leading to thrombosis invading the lymphatics and veins. These thrombosis causes emboli and vascular obstruction with the consequence of necrosis. ${ }^{8}$ The infection progressively spreads from the nasal mucosa and paranasal sinuses to the palate, orbits and brain. The orbital involvement is related to the vascular tropism of the fungus which induces vessel thrombosis, mechanical and toxic damage and subsequent tissue necrosis. ${ }^{1,6,8}$ The clinical presentation is usually unilateral, characterized with orbital 
pain, diplopia ophthalmoplegia and periorbital edema. Proptosis and even blindness, where fundoscopy is feasible central retinal vein occulusion (CRVO) or central retinal artery occulusion (CRAO) may provide a clue to the diagnosis. When any sinus disease with Apex Orbital Syndrome (AOS) is observed in a diabetic or immunocompromised patient, there should be a high index of suspicion of ROCM. Image testing are effective technique in diagnosing OAS but the most reliable is the biopsy.

Amphotericin B is the gold standard in the systemic treatment of mucormycosis. Before the use of amphotericin B in management of mucomycosis, the survival rate was only $6 \%$ whereas after the introduction of amphotericin $\mathrm{B}$, the rate dramatically increase to $60 \%$. ${ }^{10,11}$

The earlier the diagnosis and initiation of the treatment of mucormycosis the better the prognosis. Patients who begin treatment within 6 days of onset of symptoms have a survival rate of $76-81 \%$ while those that commence treatment after more than 12 days the rate reduces to $36-42 \%$.

In this index case, amphotericin was commenced 11 days after the commencement of his symptoms. This had to be discontinued due to adverse drug reaction. Posacorazole is a good alternative but was not available in this environment. This posed a great challenge in the management of this case since we had to rely on other less effective medications.

Some reports have recorded total remission of the mucormycosis on treatment with both liposomal IV amphotericin B and lavage of affected tissues with amphotericin B. Liposomal amphotericin B is the first drug of choice of treatment because it permeates the blood brain barrier more effectively. ${ }^{10,12}$

In a large European study fluconazole was ineffective while itracornazole was found to be partially effective and posacorazole was effective. . $^{13,14,15,16}$

\section{CONCLUSION}

An early diagnosis and commencement of treatment of mucormycosis is crucial for a good prognosis. When any sinus disease with Apex Orbital Syndrome (AOS) is observed in a diabetic or immunocompromised patient, there should be a high index of suspicion of ROCM.

The therapeutic challenges that confronted us in the management of this case was the patient's adverse drug reaction to the readily available and effective antifungal drug amphotericin B and the non availability of the alternatives in our environment. There is a critical need to provide essential antifungal agents when indicated.

The management of mucormycosis demands a multidisciplinary approach and there is a dire need to increase the awareness of clinicians regarding mucormycosis in Diabetic patients.

\section{Declaration of Patient Consent}

The authors certify that they have obtained all appropriate patient consent forms. In the form the patient's relative (spouse) has given her consent for the images and other clinical information to be reported in the journal. The patient's relative understands that the names and initials will not be published and due efforts will be made to conceal patient's identity, but anonymity cannot be guaranteed.

\section{Ethical Clearance}

Clearance was obtained from the Benue State University Teaching Hospital's Ethical Clearance Committee for the publication of this case report.

\section{Conflict of Interest}

None declared.

\section{REFERENCES}

1. Saegeman V, Maerterns J, Ectors N, Meersseman W, Lagrou K. Epidemiology of mucormycosis: review of 18 cases in a tertiary care hospital. Med Mycol. 2010; 48: 245-254.

2 Prabhu RM, Patel R. Mucormycosis and entomophthoramycosis: a review of the clinical manifestations, diagnosis and treatment. Clin Microbiol infect. 2004; 10:31-47.

3. Spellberg B, Ibrahim AS. Recent advances in the treatment of mucormycosis. Curr Infect Dis Rep. 2010; 12:423429.

4. Anders UM, Taylor EJ, Martel JR, Martel JB. Acute orbital apex syndrome and rhino-orbito-cerebral mucormycosis. International Medical Case Reports Journal. 2015; 8: 93 96.

5. Plowes Hernandez O, Prado Calleros HM, Soberon Marmissolle Daguerre GS, Sadek Gonzalez A. RhinoOrbito-Cerebral Mucormycosis. Management Strategies to Avoid or Limit Intracraneal Affection and Improve Survival. Acta Otorrinolaringol Esp. 2015; 66: 348-352.

6. Vironneau P, Verillaud B, Tran H, Altabaa K, Blancal JP, Sauvaget $\mathrm{E}$, et al. Rhino-orbito-cerebral mucormycosis, 
surgical treatment, state of the art. Med Sci (Paris). 2013;

29:31-35.

7. Roden MM, Zaoutis TE, Buchanan WL, Knudsen TA, Sarkisova TA, Schaufele RL, et al. Epidemiology and outcome of zygomicosis: a review of 929 reported cases. Clin Infect Dis. 2005; 41:634-653.

8. Yohai RA, Bullock JD, Aziz AA, Markert RJ. Survival factors in rhino-orbital-cerebral mucormycosis. Surv Ophthalmol. 1994; 39: 3-22.

9. Ferry AP, Abedi S. Diagnosis and Management of rhinoorbito-cerebral mucormycosis (Phycomycosis). A report of 16 personally observed cases. Ophthalmology. 1993; 90:1096-1104.

10. Petrikkos GL, Lipid Formulations of amphotericin B as first-line treatment of zygomycosis. Clin Microbiol Infect. 2009; 15:87-92

11. Ibrahim AS, Avanessian V, Spellberg B, Edward JE Jr. Liposomal amphotericin B, and not amphotericin B deoxycholate, improves survival of diabetic mice infected with Rhizopus oryzae. Antimicrob Agents Chemother. 2003; 47: 3343-3344

12. Fisher EW, Toma A, Fisher PH, Cheesman AD. Rhinocerebral mucormycosis: use of liposomal amphotericin B. J Laryngo Otol. 1991; 105:575-577

13. Ugurlu SK, Selim S, Kopar A. Rhino-orbital mucormycosis: Clinical findings and treatment outcomes of four cases. Turk J Ophthalmol 2015; 45:169-174

14. Dannauoui E, Melatiadis J, Mouton JW, Meis JF, Verweij PE. Eurofung Network. In vitro susceptibilities of zygomycetes to conventional antifungals. J Antimicrob Chemother. 2003; 51:45-52

15. Cornely OA, Vehreschild JJ, Riiping MJ. Current experience in treating invasive zygomycosis with posaconazole. Clin Microbiol Infec. 2009; 15:77-81.

16. Van Burik JA, Hare RS, Solomon HF, Corrado ML, Kontoyiannis DP. Posaconazole is effective as salvage therapy for zygomycosis. Antimicrob Agents Chemother 2006; 50:126-133. 